\title{
ISCHEMIC STROKE AS THE INITIAL MANIFESTATION OF ANTIPHOSPHOLIPID SYNDROME ASSOCIATED WITH LIBMAN SACKS ENDOCARDITIS
}

\author{
Olga Cassol Silva ${ }^{1, \star}$, Gladys Lentz Martins ${ }^{2}$, Alessandra Laitart² \\ 1.Universidade do Sul da Santa Catarina, Palhoça (SC), Brazil. 2.Hospital Governador Celso Ramos, Florianópolis (SC), Brazil. \\ *Corresponding author: olgacassol@hotmail.com
}

\section{BACKGROUND}

Antiphospholipid antibody syndrome (APS) is a rheumatological disease manifested by a hypercoagulability state. Antiphospholipid antibodies are present in $13 \%$ of patients with ischemic stroke, which is the most prevalent arterial manifestation of APS. LibmanSacks endocarditis (LSE), although rare, represents a cardiac manifestation of APS. It is estimated that $33 \%$ of patients with ELS have positive aPL.

\section{CASE REPORT}

A 24-year-old female was admitted due to an acute onset of transcortical motor aphasia that started four hours before hospital admission, without other focal neurological deficits. The general physical examination was normal. She was previously obese (grade II), with a history of pre-eclampsia followed by abortion 1 year earlier (G1P0A1). She denied using continuous medications. Laboratory tests on admission showed thrombocytopenia (60,000), high D-dimer (1,750), slightly elevated ESR (12), negative serology for syphilis, HIV, Hepatitis B and C. The computed tomography scan of the skull with contrast showed an acute left ischemic frontalopercular penumbra and large vessel occlusion was not seen. National Institutes of Health Stroke Scale (NIHSS) on the admission was three (she missed month, age and had mild aphasia). Despite the borderline T delta, thrombolytic treatment was chosen because of the aphasia and patient did not have contraindications for the procedure. The treatment was performed without complications and there was complete improvement of the aphasia after two days of hospitalization. During the etiologic investigation of stroke, it was found positive for alpha 1 glycoprotein antibodies, lupus anticoagulant and ANA positive 1/320 with a fine speckled nuclear pattern, indicative of APS. Other specific systemic lupus erythematosus antibodies were negative. Transthoracic echocardiogram was performed and identified an isoechogenic mobile polypoid image, adhered to the atrial surface in the end of the posterior mitral cusp tip, measuring $0.7 \times 0.6 \mathrm{~cm}$, suggestive of LSE. Blood cultures for typical and atypical germs were negative and the absence of fever or infectious signs during the period allowed to rule out infective endocarditis. After receiving the cardiac surgery department impression, it was decided not to perform surgery and to start anticoagulation with warfarin. The patient was discharged with NIHSS of zero and follow-up with neurology, rheumatology and cardiology with imaging control.

\section{CONCLUSION}

Although infrequent, LSE is associated with antiphospholipid antibodies. Once stroke can be due to both APS and LSE, it is reinforced the importance of careful investigation in these patients, in order to establish adequate diagnosis and therapy, avoiding such outcome. 